\title{
SUPERLUMINAL MOTIONS: THE FIRST 100 SOURCES
}

\author{
R.C. VERMEULEN \\ California Institute of Technology, \\ Astronomy 105-24, Pasadena, CA 91125, USA
}

\begin{abstract}
First results from a large homogeneous superluminal motion survey are presented. The data do not show compelling evidence for the existence of intrinsically different populations of galaxies, BL Lac objects, or quasars. $\beta_{\text {app }}$ in the range $1-5 h^{-1}$ occur with roughly equal frequency; higher values, up to $\beta_{\text {app }}=10 h^{-1}$, are rather more scarce than appeared to be the case from earlier work, which evidently concentrated on sources which are not representative of the general population. The $\beta_{\text {app }}$ distribution suggests that there might be a skewed distribution of Lorentz factors over the sample, with a peak at $\gamma_{\mathrm{b}} \approx 2 h^{-1}$ and a tail up to at least $\gamma_{\mathrm{b}} \approx 10 h^{-1}$. There appears to be a clearly rising upper envelope to the $\beta_{\text {app }}$ distribution when plotted as a function of observed $5 \mathrm{GHz}$ luminosity; a combination of source counts and the apparent velocity statistics in a larger sample could provide much insight into the properties of radio jet sources.
\end{abstract}

\section{Introduction}

This review has been adapted from a presentation first given at the conference on "High Resolution Radio Imaging of Quasars and AGN" (Vermeulen 1995). It emphasizes statistics. Some other contributions to this Symposium deal with the wealth of data available for a select few sources. For example, in 3C 345 (Zensus 1996) prolonged, intensive monitoring suggests that in the inner parsecs the jet has a constant Lorentz-factor $(\gamma \sim 10)$, but bends away from the line-of-sight by a few degrees (e.g. Zensus et al. 1995).

In contrast, the measurements presented here are mostly rather sparse. But, as long as the source selection criteria are well known, a statistical approach has the virtue that population models can be constructed, involving 
a distribution of Lorentz factors, jet bending, pattern motions, acceleration, or whatever other complexity is thought to be indicated by the data. These models can be compared to the observed distribution of (superluminal) apparent velocities with the help of Monte Carlo simulations. Thus, the apparent velocity statistics of the sample reveal for the population as a whole complexities which are missed in individual objects.

Friedmann cosmology with $H_{0}=100 \mathrm{~km} \mathrm{~s}^{-1} \mathrm{Mpc}^{-1}$ is used throughout this review, and where appropriate the dependence of the results on $h$ and $q_{0}$ is shown. Indeed, since superluminal radio sources can be observed over a wide range of redshifts, their statistics can contribute to a discrimination between different cosmological models (e.g. Cohen et al. 1988, Vermeulen \& Cohen 1994). Furthermore, if observable, individual two-sided relativistic jets near the plane of the sky would have a "standard velocity" of $2 c$, so that $h$ follows directly from the observed angular separation rate. This promise might be fulfilled by a recently discovered CSO, 1946+708 (Taylor, Vermeulen, \& Pearson 1995).

\section{Randomly Oriented and Beamed Samples; Unification Tests}

The model predictions in this review, based mostly on Monte Carlo simulations, follow the formalism and assumptions outlined earlier (Vermeulen \& Cohen 1994). If a sample has randomly oriented jets, then many will be pointed near the plane of the sky, and have $\beta_{\mathrm{app}}=v_{\mathrm{app}} / c \approx 1$. There will be a modest fraction of superluminals, from jets at angles of $\sin \theta \sim 1 / \gamma$, and a small percentage of knots with $\beta_{\text {app }}<1$, in jets pointed almost straight towards us. If, on the other hand, a flux-limited sample is selected on beamed radiation (such as flat-spectrum centimeter radio emission), then most of the observed motions should be close to the maximum possible velocity, $\beta_{\text {app }}=\beta \gamma$, because the width of the beaming cone is similar to the angle at which the largest apparent motion occurs.

The distribution of velocities in a sample is therefore potentially an indicator of the range of angles to the line-of-sight, useful to test unification models, subject to some caveats (e.g. Vermeulen \& Cohen 1994). In quasars selected at low frequency (to minimize orientation bias, see Hough 1994, and Zensus \& Porcas 1987), the apparent velocities tend decrease with decreasing core dominance ( $R$, which is thought to be another orientation indicator, e.g. Orr \& Browne 1982). However, there is significant scatter (e.g. Vermeulen et al. 1993).

Taking all data from the literature (an inhomogeneous collection, Vermeulen \& Cohen 1994), it seems that at the very largest $R$, which often occur in BL Lacs, the apparent velocities may decrease again, as expected for jets viewed within the $1 / \gamma$ beaming cone. The $\beta_{\text {app }}$ statistics presently 


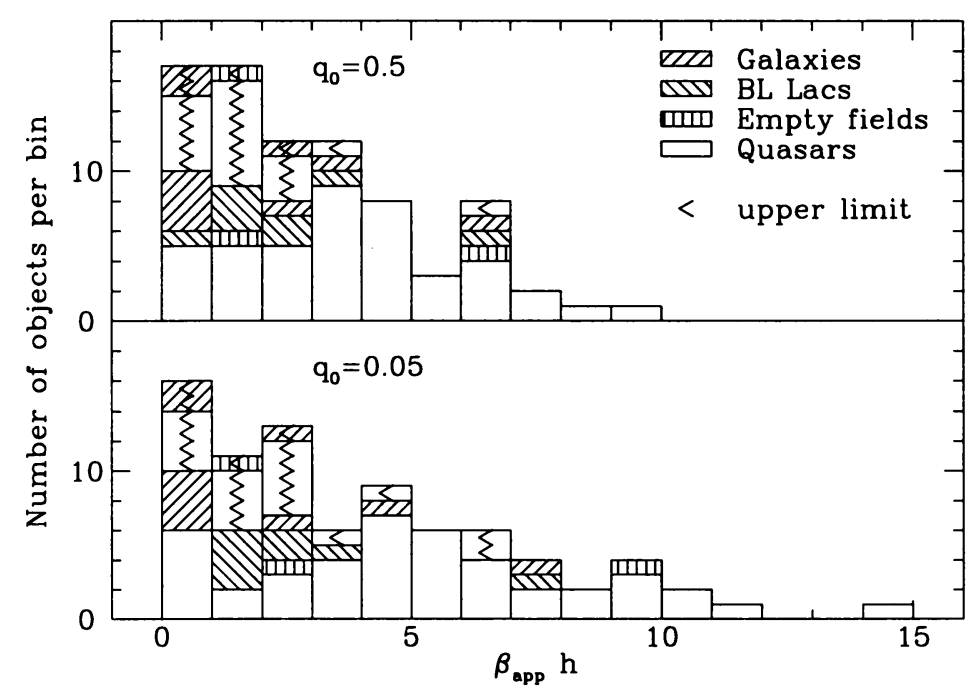

Figure 1. The observed apparent velocity distribution for 81 objects in the homogeneous $\mathrm{PR}+\mathrm{CJ}$ flat-spectrum sample, showing the scarcity of higher values, compared to earlier work and to predictions for beamed samples. There is no firm evidence for differences related to optical identification.

available do not require the BL Lacs to be intrinsically different objects, as is sometimes suggested (e.g. Gabuzda et al. 1994,Urry \& Padovani 1995), but more work on homogeneous samples is needed.

\section{First Results from the CJ Survey}

The combination of the Pearson-Readhead (Pearson \& Readhead 1988) and Caltech-Jodrell Bank VLBI surveys (Polatidis et al. 1995, Taylor et al. 1994) yields a complete flux-limited sample of 293 flat-spectrum sources brighter than $0.35 \mathrm{Jy}$ at $5 \mathrm{GHz}$. Second epoch observations, with a time interval of 2-3 years, are in progress, and will hopefully be completed in 1995. Apparent velocities or useful upper limits are now available for 81 of these sources; they are shown in Figure 1.

Ignoring upper limits and empty fields, the mean apparent velocity is slightly smaller for the galaxies $\left(N=7,\left\langle\beta_{\text {app }}\right\rangle=2.1\right)$ and the BL Lacs $\left(N=8,\left\langle\beta_{\text {app }}\right\rangle=2.3\right)$ than for the quasars $\left(N=44,\left\langle\beta_{\text {app }}\right\rangle=3.2\right)$; all for $q_{0}=0.5$. This is in the sense expected for unification models. However, the KS test yields a probability of $23 \%$ that the galaxies and the quasars have the same $\beta_{\text {app }}$ distribution, and a probability of $30 \%$ for the BL Lac objects and the quasars. With the full sample, stronger tests of unification models will be possible.

There is a substantial fraction $(\sim 25 \%)$ of stationary features or upper 
limits. Then, if $q_{0}=0.5$ it seems that the majority of $\beta_{\text {app }}$ are evenly spread over the range $1-5 h^{-1}$, with a tail $(17 \%)$ of higher values, up to $10 h^{-1}$. If $q_{0}=0.05$, the $\beta_{\text {app }}$ distribution tapers off even more gently; most values are still below $10 h^{-1}$. The fraction of sources with $\beta_{\mathrm{app}}=5-10 h^{-1}$ in the older literature (Vermeulen \& Cohen 1994) was more than twice as high, $36 \%$. The scarcity in CJ of these somewhat faster motions is not caused by undersampling in time. It seems that there used to a bias towards studying and publishing fast superluminals. A lot of the sources previously studied are highly variable (many appear in the Variable Source Sample defined by Wehrle et al. 1992, for example), so this is further evidence for a correlation between variability at high frequency and a large apparent velocity (e.g. Teräsranta \& Valtaoja 1994).

Setting aside the upper limits, and adopting $q_{0}=0.5$ (the least extreme case), the $\beta_{\text {app }}$ distribution can be reproduced if there is a wide range of Lorentz factors in the sample, with in particular a long tail to high values. As a numerical example for illustrative purposes only, if $h=0.55$ then $\gamma_{\mathrm{b}} \approx 4$ could be the peak of a skewed distribution spanning the range $\gamma_{\mathrm{b}} \approx 2-18$. Interestingly, such a distribution is akin to that derived from largély independent data, involving radio source counts (Padovani \& Urry 1992).

Well-fitting models can also be found without such a broad range of bulk Lorentz factors $\left(\gamma_{\mathbf{b}}\right)$, but assuming that the observed features are patterns with a $\gamma_{\mathrm{p}} \neq \gamma_{\mathrm{b}}$. This breaks the match between the (bulk) beaming cone and the angle at which the maximum (pattern) velocity occurs, thus yielding a larger fraction of relatively slower motions. The predicted velocity statistics are degenerate between slow and fast patterns, but this can be resolved by taking into account other measurements of the bulk Doppler factor, such as provided by the inverse-Compton X-ray deficit (Ghisellini et al. 1993) or the equipartition Doppler factor (Readhead 1994, Güijosa \& Daly 1996).

To fit the data with $q_{0}=0.5$ one would need either $r=\gamma_{\mathrm{p}} / \gamma_{\mathrm{b}} \approx 0.25$ or $r \approx 10.0$; intermediate values are ruled out, unless there is also a considerable range of $\gamma_{\mathrm{b}}$ values. Such low or high $r$ are un-appealing; $r=0.25$ because, since $\beta_{\text {app }}$ up to $10 h^{-1}$ occur, it requires a rather high $\gamma_{\mathrm{b}} \geq 40 h^{-1}$ in all objects; $r=10$ because, conversely, it requires that almost all objects have $\gamma_{\mathrm{b}} \leq 1 h^{-1}$, in contradiction with other evidence for substantial Doppler beaming. It seems that this high $r$ case is akin to an incarnation of the light-echo models (Ekers \& Liang 1990), in which relativistic motion is admitted, but Doppler beaming is not. While it is entirely plausible that pattern velocities do play a role, for example in causing apparently stationary patterns in relativistic jets, further evidence that Doppler beaming is in fact important is given by the observed luminosity dependence of the $\beta_{\text {app }}$ distribution. 


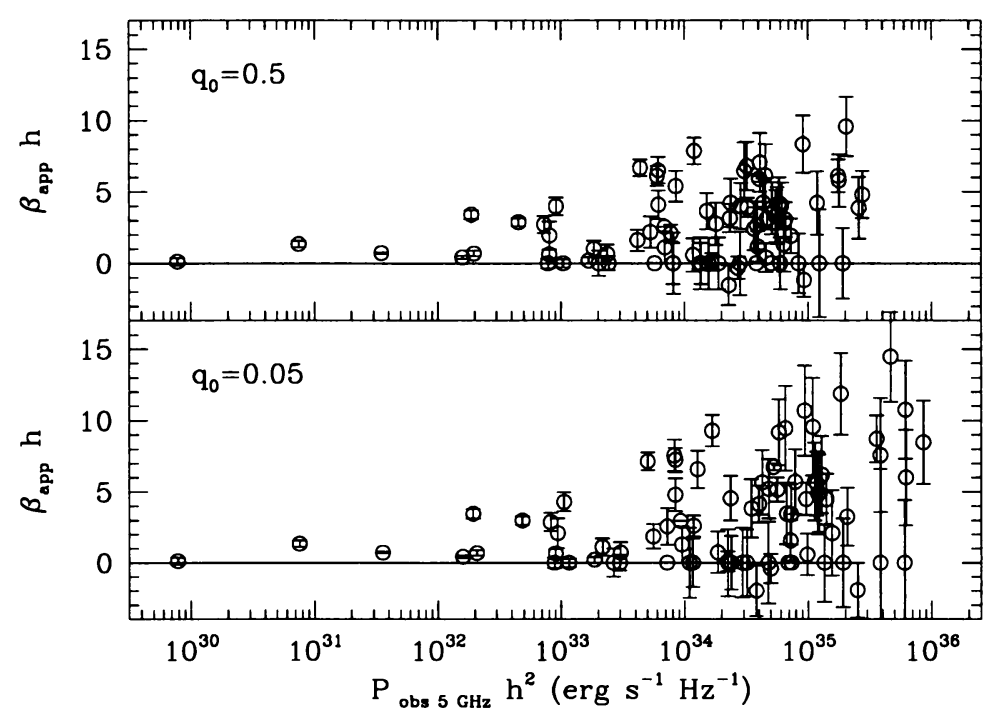

Figure 2. The observed apparent velocity distribution for 81 objects in the homogeneous $\mathrm{PR}+\mathrm{CJ}$ flat-spectrum sample, illustrating that the upper envelope rises as a function of the observed $5 \mathrm{GHz}$ monochromatic luminosity. Upper limits are plotted as error bars on $\beta_{\mathrm{app}}=0$.

Figure 2 shows the dependence of the observed $\beta_{\text {app }}$ on observed monochromatic luminosity $P_{5, \mathrm{obs}}$, calculated assuming isotropic emission. While low $\beta_{\text {app }}$ can be found at any observed luminosity, there seems to be a striking correlation of the largest $\beta_{\text {app }}$ with observed luminosity: the upper envelope rises considerably. Most of our flat-spectrum sources at the high observed luminosity end $\left(P_{5, \text { obs }} \sim 10^{35} h^{-2} \mathrm{erg} \mathrm{s}^{-1} \mathrm{~Hz}^{-1}\right)$ seem to conform roughly to the simple model: they have highly relativistic jets, and are in our sample because their observed luminosity is considerably enhanced by Doppler beaming; most of them are from a parent population 2 or 3 orders of magnitude down in intrinsic (isotropic) luminosity. But furthermore, the absence of similarly high $\beta_{\text {app }}$ at $P_{5, \text { obs }} \leq 10^{33} h^{-2} \mathrm{erg} \mathrm{s}^{-1} \mathrm{~Hz}^{-1}$ suggests that those objects are rather less beamed, which in turn implies that there is no substantial population down another 2 or 3 orders of magnitude in intrinsic luminosity, from which members can get Doppler beamed up. Thus, it would seem that highly relativistic jets may occur only in objects with a restricted range of $5 \mathrm{GHz}$ intrinsic luminosities, perhaps predominantly near $10^{32} h^{-2} \mathrm{erg} \mathrm{s}^{-1} \mathrm{~Hz}^{-1}$, and that there is a correlation between intrinsic $5 \mathrm{GHz}$ radio luminosity and Lorentz factor, akin to that often postulated for the low frequency radio luminosity (e.g. the FR-I-FR-II division). Clearly, further analysis of the effects seen in Figure 2 is needed, and a combination of source counts and the apparent velocity statistics of a larger sample could provide much insight into the properties of radio jet sources. 
Acknowledgements. The material in this paper was drawn from previous reviews (Vermeulen 1995, 1996), and updates earlier work (Vermeulen \& Cohen 1994), by including many new measurements of Caltech-Jodrell survey sources (Polatidis et al. 1995, Taylor et al. 1994), which will be published separately after further analysis. I thank all of my collaborators for their indispensable contributions, and Matthew Lister for pointing out an error in the original manuscript. This survey has been supported in part by the National Science Foundation under grants AST 88-14554, AST 91-17100, and AST 94-20018. Support to attend IAU Symposium 175 was provided by the American Astronomical Society and the International Astronomical Union.

\section{References}

Cohen, M. H., Barthel, P. D., Pearson, T. J., \& Zensus, J. A. 1988 Ap. J., 329, 1.

Ekers, R. D., \& Liang, H. 1990, in Parsec-Scale Radio Jets, eds. Zensus, J. A., \& Pearson, T. J. (Cambridge University Press: Cambridge), p. 333.

Gabuzda, D. C., Mullan, C. M., Cawthorne, T. V., Wardle, J. F. C., \& Roberts, D. H. 1994 Ap. J., 435, 140.

Ghisellini, G., Padovani, P., Celotti, A., \& Maraschi, L. 1993 Ap. J., 407, 65.

Güijosa, A., \& Daly, R. A. 1996, Ap. J., in the press.

Hough, D. H. 1994, in Compact Extragalactic Radio Sources, eds. Zensus, J. A., \& Kellermann, K. I. (NRAO: Charlottesville), p. 169.

Orr, M. J. L., \& Browne, I. W. A. 1982 Mon. Not. Royal Astron. Soc., 200, 1067.

Padovani, P., \& Urry, C. M. 1992 Ap. J., 387, 449.

Pearson, T. J., \& Readhead, A. C. S. 1988 Ap. J., 328, 114.

Polatidis, A. G., Wilkinson, P. N., Xu, W., Readhead, A. C. S., Pearson, T. J., Taylor, G. B., \& Vermeulen, R. C. 1995 ApJS 98, 1

Readhead, A. C. S. 1994 Ap. J., 426, 51.

Taylor, G. B., Vermeulen, R. C., Pearson, T. J., Readhead, A. C. S., Henstock, D. R., Browne, I. W. A., \& Wilkinson, P. N. 1994 Ap. J. Suppl., 95, 345.

Taylor, G. B., Vermeulen, R. C., \& Pearson, T. J. 1995 Proc. Natl. Acad. Sci. USA, 92, 11381.

Teräsranta, H., \& Valtaoja, E. 1994 Astron. Astrophys., 283, 51.

Urry, C. M, \& Padovani, P. 1995 Publ. Astron. Soc. Pac., 107, 803.

Vermeulen, R. C. 1995 Proc. Natl. Acad. Sci. USA, 92, 11385.

Vermeulen, R. C. 1996, in Energy Transport in Radio Galaxies and Quasars, eds. P. Hardee, A. Bridle, \& A. Zensus (Astronomical Socity of the Pacific: San Francisco), in the press.

Vermeulen, R. C. \& Cohen, M. H. 1994 Ap. J., 430, 467.

Vermeulen, R. C., Bernstein, R. A., Hough, D. H., \& Readhead, A. C. S. 1993 Ap. J., 417, 541 .

Wehrle, A. E., Cohen, M. H., Unwin, S. C., Aller, H. D., Aller, M. F., \& Nicolson, G. 1992 Ap. J., 391, 589.

Zensus, J. A. 1996, in Extragalactic Radio Sources, proc. IAU Symp. 175, ed. C. Fanti (Kluwer: Dordrecht), in the press (this volume).

Zensus, J. A., \& Porcas, R. W. 1987, in Superluminal Radio Sources, eds. Zensus, J. A., \& Pearson, T. J. (Cambridge University Press: Cambridge), p. 126.

Zensus, J. A., Cohen, M. H., \& Unwin S. C. 1995 Ap. J., 443, 35. 